\title{
Fibromyalgia and the old dilemma: theory vs. practice
}

\author{
José Eduardo Martinez \\ Pontifícia Universidade Católica de São Paulo, São Paulo, SP, Brazil
}

\begin{abstract}
The objective of this article is to review some aspects of the fibromyalgia syndrome and its mechanisms of development. We also discuss how to go from preclinical research to clinical practice. Fibromyalgia is a clinical syndrome whose main features include diffuse musculoskeletal pain, fatigue, sleep disturbances, and cognitive disorders. Russell \& Larsson (2009) proposed "pronociceptive" and "antinociceptive" systems under normal conditions. Functional pain states such as fibromyalgia are derived from central disturbances in pain processing. The association with anxiety and depression is a negative prognostic factor. Distress is an important part of its physiopathology. The concomitance of other functional syndromes is a rule. The already known etiopathogenic mechanisms of fibromyalgia can be applied in clinical practice for diagnosis and rational therapeutic approaches. Pharmacological but mainly nonpharmacological measures must be applied. Although much information still needs to be obtained, the clinician presently has sufficient tools for efficiently treating fibromyalgia patients. An important aspect is that such knowledge needs to reach primary care physicians because the prevalence of fibromyalgia does not allow all patients to be treated by specialists. Keywords: fibromyalgia, pain, central sensitization, treatment.
\end{abstract}

Received 30 January 2012; received in revised from 24 May 2012; accepted 22 August 2013. Available online 07 March 2014.

\section{Introduction}

After four decades of research on fibromyalgia, much knowledge has been acquired, but a large gap still needs to be filled. The main challenge is to link the wellestablished points and determine how clinical practice can benefit from them. The complexity of the clinical picture of fibromyalgia makes it difficult to apply the already known mechanisms of the disease to therapeutic approaches. This complexity is derived from the association between the main features of this intriguing malady to other functional clinical syndromes, psychiatric diseases, and possibly overlapping conditions. There are reasons to be optimistic because valuable research results have allowed the establishment of guidelines that involve pharmacological and nonpharmacological interventions.

The objective of this article is to review some aspects of the well-known features of fibromyalgia and its mechanisms of development. We also discuss how to go from preclinical research to clinical practice.

The most recent concept states that fibromyalgia is a clinical syndrome whose main features include diffuse musculoskeletal pain, fatigue, sleep disturbances, and cognitive disorders. These are considered the essential characteristics for recognizing this syndrome. Diffuse

José Eduardo Martinez, Department of Medicine, Pontifícia Universidade Católica de São Paulo, São Paulo, SP, Brazil. Correspondence regarding this article should be directed to: José Eduardo Martinez, Rua Portugal, 63, Sorocaba, SP, CEP 18045-280, Brazil. Phone: +55 (15) 32129879. E-mail: jemartinez@pucsp.br and amplified pain is a prerequisite for its diagnosis and the distinguishing feature between fibromyalgia and other similar conditions such as regional myosfascial pain and fatigue syndrome. We discuss not only these core symptoms but also other overlapping conditions that comprise the fibromyalgia syndrome.

\section{Pain}

Pain is always a great challenge in clinical practice, independent of its type or origin. This is particularly true for fibromyalgia in which the central issue is not simply pain but also the abnormal presentation of it. This type of pain does not fit any definition of lesion or isolated functional disturbance.

To better understand this condition, beginning with the most comprehensible situation is important. From a clinical point of view, nociceptive pain is a well-known condition. A harmful event stimulates nociceptive receptors that activate a series of events. The final consequence is pain perception.

Russell \& Larsson (2009) proposed a model in which pain perception processes can be divided into "pronociception" and "antinociception" under normal conditions. Pronociception is initiated with peripheral pain receptor activation by a mechanical, chemical, or thermal stimulus. This first step generates action potentials. After passing through a series of sensory-discriminative ascending pathways, the action potentials reach the brain and determine pain perception after synaptic contact with brainstem structures, limbic structures, the dorsal thalamus, and the neocortex (Russell \& Larsson, 2009). The main neurotransmitters 
of this system are glutamate and substance P. When coming from the brain and brainstem and descending to the dorsal horn of the spinal cord, inhibitory pathways become involved in the modulation of pain (Basbaum \& Fields, 1978; Fields \& Basbaum, 1989). At this point, serotonin, norepinephrine, dopamine, and endogenous opioid peptides are key chemical mediators. Normal pain is the result of the proper functioning of these two hodological mechanisms in the physiology of pain (Russell \& Larson, 2009). Although simplistic, this model facilitates our understanding of normal nociception and nociceptive pain.

How is this pain clinically manifested? Patients frequently not only describe its characteristics but also associate it with triggering events. The intensity of pain generally responds well to analgesic and antiinflammatory drugs (Ablin \& Clauw, 2009; Fitzcharles, Almahrezi, \& Shir, 2005). Behavioral changes occur as a consequence of pain (Ablin \& Clauw, 2009). Physicians can easily correlate pain symptoms with causes. Pain has a reason to exist and protects the organism from harm.

However, two other painful conditions do not fit this explanation: (1) when pain transmission structures are harmed in what is called neuropathic pain and (2) when central sensitization characterizes a dysfunctional state such as the one that occurs in the fibromyalgia syndrome (Ablin \& Clauw, 2009; Galvão, 2005).

Neuropathic pain may derive from infectious (herpes zoster), metabolic (diabetes mellitus), and traumatic events that cause temporary or permanent functional disturbances or structural lesions in peripheral or central neural pathways (Galvão, 2005). In addition to intense pain, the other main clinical features include paresthesias and alterations in sensitivity (Galvão, 2005). Hyperalgesia, allodynia, and neurogenic inflammation are also observed. These are characterized by neuroplastic phenomena that promote the maintenance of pain symptoms (Ablin \& Clauw, 2009).

Abnormal pain states are derived from central disturbances in pain processing (Russell \& Larson, 2009; Ablin \& Clauw, 2009) including fibromyalgia syndrome, tension headache, and myofascial regional pain. In addition to diffuse spontaneous musculoskeletal pain, it also presents with hyperalgesia and allodynia, which characterize the pain amplification process. One important feature is that it can be superimposed on other functional syndromes characterized by central hypersensitivity, such as irritable bowel syndrome, premenstrual tension syndrome, and migraine. Other important aspects to be considered include the frequent concomitance with anxiety and depression and abnormal behavioral and physical responses to stress-related stimuli (Russell \& Larson, 2009; Ablin \& Clauw, 2009).

\section{Fibromyalgia syndrome: core symptoms}

Diffuse musculoskeletal pain is a prerequisite for the diagnosis of fibromyalgia for every classification or diagnostic criteria published for this syndrome to date (Ablin \& Clauw, 2009). Disturbances in pain perception processing are considered the central disease mechanism. Altered pain perception is caused by an imbalance between ascending nociceptive pathway activity (pronociception) and the functioning of the descending modulation system or analgesic mechanisms of the dorsal horn of the spinal cord (antinociception). It is clinically manifested by diffuse spontaneous musculoskeletal pain, hyperalgesia, and allodynia (Russell \& Larson, 2009).

But how can one explain the other cardinal features? Fatigue, sleep disturbances, and cognitive alterations are almost always present. These symptoms can sometimes dominate the clinical picture.

Fatigue may be considered a symptom associated with high sensitivity but low specificity (Arnold, 2008). Fibromyalgia is always considered a possible diagnosis when both symptoms (i.e., pain and fatigue) come together. The physician should be very mindful to assess fatigue. Arnold (2008) stated that fatigue may masquerade as a series of other symptoms with different natures: (1) physical (e.g., reduced activity, low energy, tiredness, decreased physical endurance, increased effort with physical tasks, difficulty overcoming inactivity, general weakness, heaviness, slowness or sluggishness, nonrestorative sleep, and sleepiness), (2) cognitive (e.g., decreased concentration, decreased attention, decreased mental endurance, and slowed thinking), and (3) emotional (e.g., decreased motivation or initiative, decreased interest, feeling overwhelmed, feeling bored, aversion to effort, and feeling low). In fact, just the presence of chronic pain can cause fatigue. In this case, it has been attributed to related features such as insomnia, muscular deconditioning, and depression (Fishbain et al., 2005).

Sleep disturbances caused by fibromyalgia are one of the most studied features as suggested by Moldofsky, Scarisbrick, England, \& Smythe (1975) and Moldofsky \& Lue (1980). According to these authors, this symptom is similar to that reported by patients diagnosed with fibromyalgia and can be induced in healthy sleep-deprived subjects. The most common electroencephalographic anomaly is an increase in alpha activity during non-rapid-eye-movement sleep. This sleep irregularity is frequently associated with disturbed sleep (Branco, Atalaia, \& Paiva, 1994; Drewes, Nielsen, Taagholt, Bjerregard, Svendsen, \& Gade, 1995).

More recently, the presence of cognitive disturbances has received attention from fibromyalgia researchers as one of the core features of the fibromyalgia syndrome. Fibromyalgia patients frequently complain of difficulties in memory, logical analysis, motivation, and concentration (Glass, 2008). These symptoms are highly correlated with pain, fatigue, and depression (Suhr, 2003). Imaging studies showed reduced gray matter volume in the postcentral gyrus, amgygdaloid complex, hippocampus superior frontal gyrus, and anterior cingulate gyrus (Lutz et al., 2008). The real meaning of these findings needs to be better understood. A possible relationship with the chronic stress response 
has been considered (Grisart, Van der Linden, \& Masquelier, 2002).

\section{Depression and anxiety}

The association with psychiatric diseases has been studied since the early definition of the syndrome. Important reasons for this interest are the similarity between some fibromyalgia symptoms and depression symptoms and the high subjectivity of its clinical characteristics. The psychiatric diagnoses most frequently associated with fibromyalgia are bipolar disorder, major depressive disorder, anxiety disorders, eating disorders, and substance use disorder. Since the beginning of fibromyalgia research, it has been considered a part of the affective disorder spectrum because of its high association with depression and anxiety (Hudson \& Pope, 1989). Another important point is that this association is a negative prognostic factor because depression and anxiety have deleterious influences on the expression of fibromyalgia (Ablin \& Clauw, 2009). The medical literature emphasizes the association with depression, but in day-to-day clinical practice, state or trait anxiety is frequently present and may be more problematic to the patient's life (Cohen, Buskila, Neumann, \& Ebstein, 2002). The association between several grades of anxiety and their negative impact on quality of life is clear (Pagano, Matsutan, Ferreira, Marques, \& Pereira, 2004).

\section{Distress}

One of the first demonstrations of the influence of an abnormal stress response on the physiopathology of the fibromyalgia syndrome was the description of its development after exposure to chronic and even acute stressors. The medical literature has attributed early life trauma, both physical and emotional, to the triggering of this clinical condition (Buskila, Neumann, Vaisberg, Alkalay, \& Wolfe, 1997; McLean \& Clauw, 2004; Clauw, 2003).

To establish the presence of alterations in the stress response, numerous studies have evaluated the hypothalamic-pituitary-adrenal axis. Most of these studies reported hyporeactivity to experimentally applied stressful stimuli. Still unknown is whether it is the cause or consequence of pain, but one can suppose that these disorders are responsible for part of the symptoms of fibromyalgia such as fatigue and cognitive disturbances (Adler \& Geenen, 2005).

With regard to the autonomic nervous system, hyperactivity of the sympathetic nervous system is more apparent at night (Martinez-Lavin, Hermosillo, Rosas, \& Soto, 1998; Raj, Brouillard, Simpson, Hopman, \& Abdollah, 2000). This finding has been attributed to a chronic state of distress.

\section{Overlapping syndromes: central sensitivity syndromes}

Since its first description, fibromyalgia has been associated with other syndromes that are considered to have a functional nature because they do not present any pathological lesions. Among these are chronic fatigue syndrome, irritable bowel syndrome, other functional gastrointestinal disorders, temporomandibular joint disorder, restless leg syndrome, periodic limb movements in sleep, idiopathic low back pain, multiple chemical sensitivity, primary dysmenorrhea, headache (tension headache more than migraine and mixed headache), migraine, interstitial cystitis/chronic prostatitis/painful bladder syndrome, chronic pelvic pain, endometriosis, myofascial pain syndrome, and regional soft tissue pain syndrome (Romano, 1988; Yunus, Masi, \& Aldag, 1989; Triadafilopoulos, Simms, \& Goldenberg, 1991; Sperber et al., 1999; Sivri, Cindas, Dincer, \& Sivri, 1996; Wolfe, Ross, Anderson, Russell, \& Hebert, 1995).

Yunus (2008) proposed that these nosological entities form a continuum that has a common pathophysiological pathway based on central nervous system hypersensitivity. He called this "Central Sensitivity Syndrome." This new clinical construct may spur new research and even a common approach to all of these conditions that considers clinical, pathophysiological, and emotional aspects prevalent in these syndromes.

Another important overlap is musculoskeletal disease, which is frequently prevalent at the same age as fibromyalgia. Most of these diseases have chronic nociceptive pain as their main symptom. Because fibromyalgia is considered a pain amplification syndrome derived from central hypersensitivity, it can be concluded that a continuous pain stimulus input may contribute to the maintenance and even worsening of pain intensity. This aspect has a significant impact on differential diagnosis and the evaluation of severity.

\section{Where are we now?}

These aforementioned considerations point to the following topics:

1 - The cardinal symptoms of fibromyalgia are diffuse pain, fatigue, sleep disturbances, and cognitive dysfunction.

2 - The mechanism of pain in fibromyalgia comprises central sensitization with enhanced pain perception derived from an imbalance between pronociceptive and inhibitory pathways.

3 - Depression and anxiety are frequently associated with fibromyalgia and may be a poor prognostic factor.

4 - Distress is an important mechanism of disease and likely responsible for the other core symptoms.

5 - Fibromyalgia is part of group of other symptoms that have central sensitivity in common. This group of syndromes shares a number of symptoms including fatigue, sleep disturbances, cognitive dysfunction, and psychiatric conditions.

6 - The presence of comorbid musculoskeletal conditions is frequent and may also be a factor for poor prognosis. 


\section{How our knowledge may be applied in clinical practice}

The first issue to be discussed is diagnosis. Fibromyalgia is composed of few symptoms and no reliable signs, and the signs and symptoms are subjective. There is also frequent overlap with other clinical conditions and the presence of several factors of poor prognosis, all of which make the diagnosis of fibromyalgia difficult. Since the beginning of the recognition of fibromyalgia when it was little recognized or even believed until now when there is apparently an excess of diagnosis, much has changed.

The standardization of diagnostic criteria for its inclusion in scientific papers was achieved with the publication of the American College of Rheumatology (ACR) 1990 Classification Criteria (Wolfe et al., 1990). A clinician shall consider as important features the role of symptoms and presence of overlapping conditions. Although this is true today, the 1990 ACR criteria have emphasized the number of tender points (Figure 1) and the presence of diffuse pain. These two characteristics are responsible for its diagnosis (Wolfe et al., 1990). This 1990 publication made it clear that the presented criteria have only classification purposes and should not be used for diagnosis. Research from then until now has reported patients who may not present the entire spectrum of fibromyalgia.

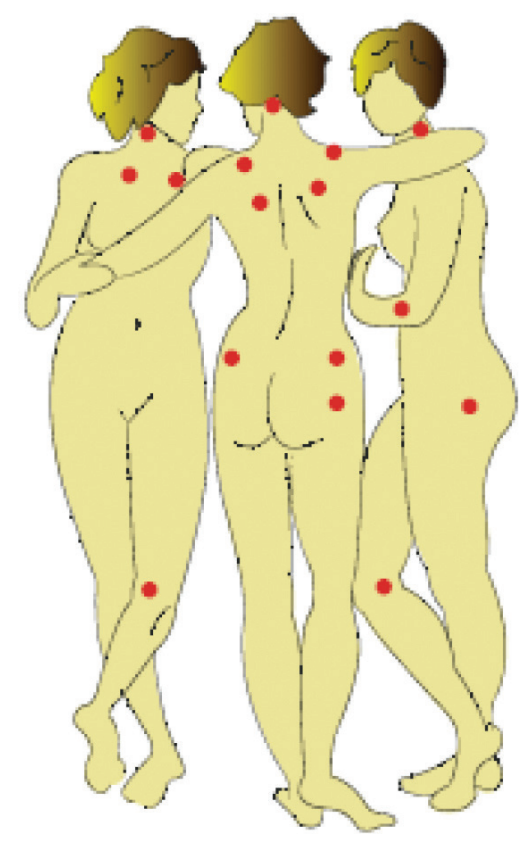

Figure 1. American College of Rheumatology Tender Point Chart.

To correct this situation, in 2010, ACR presented preliminary diagnostic criteria to complement clinical investigations, which were then modified in 2011 (Wolfe et al., 2010, 2011). According to these new criteria, not only pain but also other cardinal symptoms were taken into account. To be sure, this appears to be an advance, but clinicians in daily practice should rely on clinical observations and their pathophysiological knowledge to make a precise diagnosis and use these diagnostic tools as a complement to traditional diagnostic methods. The use of these diagnostic criteria also benefits patient follow-up and epidemiological studies.

Modified from American College of Rheumatology diagnostic criteria.

Criteria: Fibromyalgia is considered when the following three conditions are met: (1) Widespread Pain Index $\geq 7$ and Symptom Severity Score $\geq 5$ or Widespread Pain Index between 3 and 6 and Symptom Severity Score $\geq 9$, (2) the symptoms must have a duration of 3 months, and (3) the patient should not have another disease that explains the pain.

Widespread Pain Index is composed of the sum of the bodily areas indicated by the patient as spontaneously painful in a presented list.

Symptom Severity Score is the sum of partial scores on three disturbances: fatigue, sleep disturbances, and cognitive symptoms. For each symptom, the following scale shall be used: $0=$ no problem, $1=$ slight or mild problem, 3 = severe, continuous, and life-disturbing problems.

It is also important to point out the necessity of a complete musculoskeletal assessment for a precise diagnosis of other possible comorbid musculoskeletal conditions that can maintain a pain input to the central nervous system. One important possible cause for treatment failure is considering that all of the pain that the patient experiences is the result of fibromyalgia.

With regard to treatment, it is imperative to address nonpharmacological approaches together with pharmacological interventions. This is because there is no single or associated pharmacological regimen that can achieve complete successful treatment. Adverse events associated with drugs must also be considered.

Health education is one of the main nonpharmacological approaches. A fully oriented patient will help adhere to therapeutic procedures. It is essential to provide information about the symptoms, the mechanisms of disease, prognosis, and therapeutic measures (Buckelew, Murray, Hewett, Johnson, \& Huyser, 1995; Nicassio et al., 1997).

Another essential therapeutic method is exercise. It is useful against several features of the disease such as pain, fatigue, sleep disturbances, depression, and some of overlapping conditions. The best scientific evidence has been obtained for supervised aerobic exercise. The addition of strength and flexibility exercises may increase efficacy in relation to functional capacity and symptom severity, but little evidence supports these 
approaches (Valim et al., 2003; Sanudo, Galiano, Carrasco, Blagojevic, de Hoyo, \& Saxton, 2010).

To address the emotional and physical aspects of psychological techniques and exercises, cognitive behavioral therapy has been used with good scientific correlates (Keefe \& Caldwell, 1997).

With regard to pharmacological management, improving pain modulation is necessary. The pronociceptive and antinociceptive model may be useful for this purpose. It is important to enhance the antinociceptive system and inhibit the pronociceptive system. To accomplish this, physicians have used medications that interfere with the neurotransmitters involved in these two functions (Russell \& Larson, 2009).

As already stated, the main inhibitory pain neurotransmitters are serotonin and norepinephrine. Both act at descending inhibitory pain pathways. These same substances are involved in other aspects of fibromyalgia syndrome such as the stress response, sleep physiology, and mood disturbances. Drugs that inhibit synaptic reuptake include tricyclic and dual antidepressants. Among these, the most studied drugs with the strongest evidence are amitriptyline (Nishishinya et al., 2008), duloxetine (Arnold et al., 2004), and milnacipran (Gendreau et al., 2005). One notable medication is cyclobenzaprine (Tofferi, Jackson, \& O'Malley, 2004), which has the same mode of action but is used for its muscle relaxant properties. The selective serotonin reuptake inhibitor fluoxetine is beneficial at doses of 10-80 mg/day (Arnold, Hess, Hudson, Welge, Berno, $\&$ Keck, 2002). Another useful drug is tramadol. It is a weak $\mu$-opioid receptor agonist, and its beneficial effect may derive not from its opioid function but from its known action on the inhibitory neurotransmitters at the synapses of the neurons responsible for pain transmission, such as neuroepinephrine and serotonin (Bennett, Karim, Karim, \& Rosenthal, 2003; Biasi, Manca, Manganelly, \& Marcolongo, 1998).

Medications that inhibit the pronociceptive system also modulate $\mathrm{Ca}^{2+}$ channel activity and decrease $\mathrm{Ca}^{2+}$ entry into the distal part of proximal neurons, thus reducing pain-stimulating synaptic neurotransmitter release. As stated above, the most important algogenic pain transmitters are glutamate and substance $P$. The most studied drugs in fibromyalgia that act on these neurotransmitters are pregabalin and gabapentin (Häuser, Bernardy, Üceyler, \& Sommer, 2009). The use of peripheral analgesics is only justified for the treatment of concomitant musculoskeletal conditions, which are very frequent.

\section{Conclusion}

Although much knowledge still needs to be attained, clinicians currently have sufficiently useful tools for assessing and treating fibromyalgia patients. In our opinion, the main reason for treatment failure is the scarcity of resources such as multidisciplinary teams and the lack of easy access to some medications by the low-income population. Another important aspect is that such knowledge must reach primary care physicians because the prevalence of fibromyalgia does not allow all patients to be treated by specialists.

\section{References}

Ablin, K., \& Clauw, D. J. (2009). From fibrositis to functional somatic syndromes to a bell-shaped curve of pain and sensory sensitivity: Evolution of a clinical construct. Rheumatic Diseases Clinics of North America, 35, 233-251.

Adler, G. K., \& Geenen, R. (2005). Hypothalamic-pituitary-adrenal and autonomic nervous system functioning in fibromyalgia. Rheumatic Diseases Clinics of North America, 31, 187-202.

Arnold, L. M. (2008). Understanding fatigue in major depressive disorder and other medical disorders. Psychosomatics, 49(3), 185190.

Arnold, L. M., Hess, E. V., Hudson, J. I., Welge, J. A., Berno, S. E., \& Keck, P. E., Jr. (2002). A randomized, placebo-controlled, doubleblind, flexible-dose study of fluoxetine in the treatment of women with fibromyalgia. American Journal of Medicine, 112, 191-197.

Arnold, L. M., Lu, Y., Crofford, L. J., Wohlreich, M., Detke, M. J., Iyengar, S., \& Goldstein, D. J. (2004). A double-blind, multicenter trial comparing duloxetine with placebo in the treatment of fibromyalgia patients with or without major depressive disorder. Arthritis and Rheumatism, 50(9), 2974-2984.

Basbaum, A. I., \& Fields, H. L. (1978). Endogenous pain control mechanisms: Review and hypothesis. Annals of Neurology, 4, 451462.

Bennett, R. M., Kamin, M., Karim, R., \& Rosenthal, N. (2003). Tramadol and acetaminophen combination tablets in the treatment of fibromyalgia pain: A double-blind, randomized, placebocontrolled study. American Journal of Medicine, 114, 537-545.

Biasi, G., Manca, S., Manganelly, S., \& Marcolongo, R. (1998). Tramadol in the fibromyalgia syndrome: A controlled clinical trial versus placebo. International Journal of Clinical Pharmacology Research, 18(1), 13-19.

Branco, J., Atalaia, A., \& Paiva T. (1994). Sleep cycles and alphadelta sleep in fibromyalgia syndrome. Journal of Rheumatology, 21, 1113-1117.

Buckelew, S. P., Murray, S. E., Hewett, J. E., Johnson, J., \& Huyser, B. (1995). Self-efficacy, pain, and physical activity among fibromyalgia subjects. Arthritis Care and Research, 8, 43-50.

Buskila, D., Neumann, L., Vaisberg, G., Alkalay, D., \& Wolfe, F. (1997). Increased rates of fibromyalgia following cervical spine injury: A controlled study of 161 cases of traumatic injury. Arthritis and Rheumatism, 40(3), 446-452.

Clauw D. (2003). The health consequences of the first Gulf War. British Medical Journal, 327, 1357-1358.

Cohen, H., Buskila, D., Neumann, L., \& Ebstein, R. P. (2002). Confirmation of an association between fibromyalgia and serotonin transporter promoter region (5-HTTLPR) polymorphism, and relationship to anxiety-related personality traits. Arthritis and Rheumatism, 46(6), 845-857.

Drewes, A. M., Nielsen, K. D., Taagholt, S. J., Bjerregard, K., Svendsen, L., \& Gade, J. (1995). Sleep intensity in fibromyalgia: Focus on the microstructure of the sleep process. British Journal of Rheumatology, 34, 629-635.

Fields, H. L., \& Basbaum A. I. (1989). Endogenous pain control mechanisms. In: P. D. Wall, \& R. Melszak (Eds.), Textbook of pain (pp. 206-217). Edinburgh: Churchill Livingston.

Fishbain, D. A., Lewis, J., Cole, B., Cutler, B., Smets, E., Rosomoff, H., \& Rosomoff, R. S. (2005). Multidisciplinary pain facility treatment outcome for pain-associated fatigue. Pain Medicine, 6(4), 299-304.

Fitzcharles, M. A,, Almahrezi, A., \& Shir, Y. (2005). Pain: Understanding and challenges for the rheumatologist. Arthritis and Rheumatism, 52, 3685-3692.

Galvão, A. C. R. (2005). Dor neuropática: Tratamento com anticonvulsivantes. São Paulo: Segmento Pharma.

Gendreau, R. M., Thorn, M. D., Gendreau, J. F., Kranzler, J. D., Ribeiro, S., Gracely, R. H., ... Clauw, D. J. (2005). Efficacy of milnacipran in patients with fibromyalgia. Journal of Rheumatology, 32, 1975 1985. 
Glass, J. M. (2008). Fibromyalgia and cognition. Journal of Clinical Psychiatry, 69(Suppl. 2), 20-24.

Grisart, J., Van der Linden, M., \& Masquelier, E. (2002). Controlled processes and automaticity in memory functioning in fibromyalgia patients: Relation with emotional distress and hypervigilance. Journal of Clinical and Experimental Neuropsychology, 24(8), 994-1009.

Häuser, W., Bernardy, K., Üceyler, N., \& Sommer, C. (2009). Treatment of fibromyalgia syndrome with gabapentin and pregabalin: A metaanalysis of randomized controlled trials. Pain, 145, 69-81.

Hudson, J. I., \& Pope, H. G., Jr. (1989). Fibromyalgia and psychopathology: Is fibromyalgia a form of "affective spectrum disorder"? Journal of Rheumatology Supplement, 19, 15-22.

Keefe, F. J., \& Caldwell, D. S. (1997). Cognitive behavioral control of arthritis pain. Medical Clinics of North America, 81, 277-290.

Lutz, J., Jager, L., de Quervain, D., Krauseneck, T., Padberg, F., Wichnalek, M., ... Schelling, G. (2008). White and gray matter abnormalities in the brain of patients with fibromyalgia: A diffusiontensor and volumetric imaging study. Arthritis and Rheumatism, 58(12), 3960-3969.

Martinez-Lavin, M., Hermosillo, A. G., Rosas, M., \& Soto, M. E. (1998). Circadian studies of autonomic nervous balance in patients with fibromyalgia: A heart rate variability analysis. Arthritis and Rheumatism, 41(11), 1966-1971.

McLean, S. A., \& Clauw, D. J. (2004). Predicting chronic symptoms after an acute "stressor": lessons learned from 3 medical conditions. Medical Hypotheses, 63(4), 653-658.

Moldofsky, H., \& Lue, F. A. (1980). The relationship of alpha and delta EEG frequencies to pain and mood in "fibrositis" patients treated with chlorpromazine and L-tryptophan. Electroencephalography and Clinical Neurophysiology, 50(1-2), 71-80.

Moldofsky, H., Scarisbrick, P., England, R., \& Smythe, H. (1975). Musculosketal symptoms and non-REM sleep disturbance in patients with "fibrositis syndrome" and healthy subjects. Psychosomatic Medicine, 37, 341-351.

Nicassio, P. M., Radojevic, V., Weisman, M. H., Schuman, C., Kim, J., Schoenfeld-Smith, K., \& Krall, T. (1997). A comparison of behavioral and educational interventions for fibromyalgia. Journal of Rheumatology, 24, 2000-2007.

Nishishinya, B., Urrutia, G., Walitt, B., Rodriguez, A., Bonfill, X., Alegre, C., \& Darko, G. (2008). Amitriptyline in the treatment of fibromyalgia: A systematic review of its efficacy. Rheumatology, 47, 1741-1746.

Pagano, T., Matsutan, L. A., Ferreira, E. A., Marques, A. P., \& Pereira, C. A. B. (2004). Assessment of anxiety and quality of life in fibromyalgia patients. São Paulo Medical Journal, 122(6), 252258.

Raj, S. R., Brouillard, D., Simpson, C. S., Hopman, W. M., \& Abdollah, H. (2000). Dysautonomia among patients with fibromyalgia: A noninvasive assessment. Journal of Rheumatology, 27(11), 26602665.

Romano, T. J. (1988). Coexistence of irritable bowel syndrome and fibromyalgia. West Virginia Medical Journal, 84(2), 16-18.
Russell, I. J., \& Larson A. A. (2009). Neurophysiopathogenesis of fibromyalgia syndrome: A unified hypothesis. Rheumatic Diseases Clinics of North America, 35, 421-435.

Sanudo, B., Galiano, D., Carrasco, L., Blagojevic, M., de Hoyo, M., \& Saxton, J. (2010). Aerobic exercise versus combined exercise therapy in women with fibromyalgia syndrome: A randomized controlled trial. Archives of Physical Medicine and Rehabilitation, 91, 1838-1843.

Sivri, A., Cindas, A., Dincer, F., \& Sivri, B. (1996). Bowel dysfunction and irritable bowel syndrome in fibromyalgia patients. Clinical Rheumatology, 15(3), 283-286.

Sperber, A. D., Atzmon, Y., Neumann L., Weisberg, I., Shalit, Y., AbuShakrah, M., ... Buskila, D. (1999). Fibromyalgia in the irritable bowel syndrome: Studies of prevalence and clinical implications. American Journal of Gastroenterology, 94(12), 3541-3546.

Suhr, J. A. (2003). Neuropsychological impairment in fibromyalgia: Relation to depression, fatigue, and pain. Journal of Psychosomatic Research, 55(4), 321-329.

Tofferi, J. K., Jackson, J. L., \& O’Malley, P. G. (2004). Treatment of fibromyalgia with cyclobenzaprine: A meta-analysis. Arthritis and Rheumatism, 51(1), 9-13.

Triadafilopoulos, G., Simms, R. W., \& Goldenberg, D. L. (1991). Bowel dysfunction in fibromyalgia syndrome. Digestive Diseases and Sciences, 36(1), 59-64.

Valim, V., Oliveira, L., Suda, A., Silva, L., de Assis, M., Barros-Neto, T., ... Natour, J. (2003). Aerobic fitness effects in fibromyalgia. Journal of Rheumatology, 30, 1060-1069.

Wolfe, F., Clauw, D. J., Fitzcharles, M. A., Goldenberg, D. L., Hauser, W., Katz, R. S., ... Winfield, J.B. (2011). Fibromyalgia criteria and severity scales for clinical and epidemiological studies: A modification of the ACR Preliminary Diagnostic Criteria for Fibromyalgia. Journal of Rheumatology, 38, 1113-1122.

Wolfe, F., Clauw, D. J., Fitzcharles, M. A., Goldenberg, D. L., Katz, R. S., Mease, P., ... Yunus, M. B. (2010). The American College of Rheumatology Preliminary Diagnostic Criteria for Fibromyalgia and Measurement of Symptom Severity. Arthritis Care and Research, 62(5), 600-610.

Wolfe, F., Ross, K., Anderson, J., Russell, I. J., \& Hebert, L. (1995). The prevalence and characteristics of fibromyalgia in the general population. Arthritis and Rheumatism, 38(1), 19-28.

Wolfe, F., Smythe, H. A., Yunus, M. B., Bennett, R. M., Bombardier, C., Goldenberg, D. L., ... Sheon, R.P. (1990). The American College of Rheumatology 1990 Criteria for the Classification of Fibromyalgia: Report of the Multicenter Criteria Commitee. Arthritis and Rheumatism, 33(2), 160-172.

Yunus, M.B. (2008). Central sensitivity syndromes: A new paradigm and group nosology for fibromyalgia and overlapping conditions, and the related issue of disease versus illness. Seminars in Arthritis and Rheumatism, 37, 339-352.

Yunus, M. B., Masi, A. T., \& Aldag, J. C. (1989). A controlled study of primary fibromyalgia syndrome: Clinical features and association with other functional syndromes. Journal of Rheumatology Supplement, 19, 62-71. 